\title{
El camino de la fórmula: el caso del uso de tiourea para limpieza de plata
}

\author{
Jannen Contreras Vargas
}

\begin{abstract}
La actitud científica es una capacidad de asombro que lleva a la búsqueda, un escepticismo necesario para cuestionar todo, incluyéndose a sí mismo. La gran diferencia que tenemos del resto de las especies es precisamente la capacidad de cuestionar, de preguntar, no sólo de sobrevivir.
\end{abstract}

Antonio Sánchez Ibarra (astrónomo)

- La restauración es una ciencia o un oficio? Cuando era estudiante, en varias ocasiones se planteó esta pregunta y recuerdo que, tras muchas discusiones, no se llegó a conclusión alguna. Tal vez la causa se encuentre en el devenir de la historia de la restauración, desde la época en que sólo se la reconocía como un oficio, hasta su actual situación profesional.

Este texto, que aborda el empleo de la tiourea como agente de limpieza de plata, ejemplifica qué tan inadecuado resulta concebir la restauración de bienes culturales como un mero oficio.

\section{¿Ciencia u oficio?}

Muchos de quienes nos dedicamos a la restauración la hemos comparado con la medicina, pero, según el Diccionario de la Lengua Española de la Real Academia (DRAE), la medicina es la "ciencia y el arte de precaver y curar las enfermedades del cuerpo humano", en tanto que a la restauración la define como "reparar, renovar o volver a poner algo en el estado o estimación que antes tenía". Parece, entonces, que no hay grandes similitudes, pero además, la definición del segundo término no se ocupa de la disciplina a la que nos dedicamos: prevenir y curar las "enfermedades" de los bienes culturales.

Oficio, por su parte, significa "profesión de algún arte mecánica", y si bien la restauración necesita un componente artesanal, no podemos delimitarla de esta forma, puesto que implicaría ubicarla como una actividad que, aunque ejercida con sumo cuidado, prioriza la habilidad manual y la aplicación de fórmulas sobre la reflexión y la resolución de problemas; esto soslaya toda la investigación, la argumentación teórica y la consideración del impacto en el inexcusable uso social del patrimonio.

Una fórmula -sigo al DRAE- es la "composición de una mezcla e instrucciones para su elaboración". Así que, como las artes mecánicas, las fórmulas son útiles; el problema es que, si las aplicamos de modo generalizado e irreflexivo, reducimos nuestro trabajo a su simple aspecto funcional, no muy diferente de lo que haría un buen panadero. 
En el diccionario citado ciencia, por su parte, está definida como el "conjunto de conocimientos obtenidos mediante la observación y el razonamiento sistemáticamente estructurados y de los que se deducen principios y leyes generales". En restauración, la observación y el razonamiento estructurados resultan indispensables, y se llega a conclusiones válidas y comprobables que eventualmente actuarán, por supuesto, como principios generales con toda la especificidad que cualquier ley general puede tener.

Por lo tanto, la restauración, como disciplina, puede considerarse, desde su definición, como una ciencia. Con base en lo aquí expuesto, propongo entender a la restauración como la ciencia y el arte de precaver y remediar las alteraciones de los bienes culturales que demeriten o impidan su uso social. Así, merece que los restauradores la asumamos no como un oficio, como ocurre con frecuencia, sino como una ciencia, pues identificamos detallada y analíticamente los deterioros en las obras, para después plantear una propuesta de intervención que suele echar mano de una serie de fórmulas conocidas. Es en este punto donde, comúnmente, sale a flote tanto la perenne presión por concluir las intervenciones como una cierta ausencia de conciencia -O una mezcla de ambas-, con el "argumento" de que en el pasado así ha funcionado y ha quedado bien, sin cuestionar mayormente los objetivos de la intervención ni el funcionamiento del tratamiento ni su eficacia o sus resultados a largo plazo.

Consciente de que nuestra profesión sólo se desarrollará cabalmente en la medida en que cuestionemos y nos cuestionemos, planteo lo que ha sido hasta ahora la aplicación y la enseñanza mecánica de una fórmula: el empleo de tiourea ácida para la limpieza de plata.

\section{La limpieza de plata}

Al intervenir bienes culturales metálicos, entre otros objetivos buscamos estabilizarlos materialmente para que, mediante la eliminación de fuentes de corrosión potenciales y productos de corrosión activos, su información y significado se transmitan a usuarios presentes y futuros.

El caso de la plata es peculiar, porque sus productos de corrosión más comunes -los sulfuros de plata-, son estables y su eliminación no es esencial para la permanencia de la obra. No obstante, esta corrosión no debiera conservarse, porque frecuentemente afecta el entendimiento de obras que fueron creadas para lucir con todo su brillo y suntuosidad. Sin embargo, esto no implica que la plata siempre ha de restaurarse para lograr un acabado resplandeciente; es necesario evaluar el uso, la intención, el significado y la historia de la obra, antes de determinar cuál será el objetivo de nuestra intervención.

Incluso algunas obras que se crearon con oscurecimientos de corrosión deliberados, para acentuar detalles y volumetría, han sido "limpiadas" -por desconocimiento de la técnica de factura y porque no existe diferencia química alguna que permita distinguir la corrosión natural de la formada intencionalmente-, eliminando sus efectos plásticos y dañando, así, su apreciación y aun su significado. Mención especial merecen los objetos de "plata oxidada", acabado que estuvo en boga desde 1840 hasta inicios del siglo XX, consistente en la formación de capas de sulfuros generalizadas que se pulín hasta obtener una apariencia lustrosa (Rudoe 1993:161), y que, debido al desconocimiento de esta técnica, han llegado a sufrir la eliminación total del acabado.

Cuando se trata de superficies de plata sobredorada, no hay lugar para la duda: imposible pensar que la corrosión sobre la capa de oro haya sido intencional. Los dorados se aplican para dar la impresión de mayor lujo y, ya que no se corroe, por razones litúrgicas e higiénicas. De tal forma, la corrosión sobre la capa de oro no sólo afecta su apreciación, sino que adultera su sentido.

Para la eliminación de productos de corrosión de plata existe una variedad de materiales de limpieza química: ácidos, algunos álcalis, agentes reductores y una gama relativamente pequeña de agentes quelantes, secuestrantes o acomplejantes, que son sustancias iónicas que, en condiciones particulares de $\mathrm{pH}$, son capaces de coordinar iones metálicos específicos para formar complejos estables. Entre estos últimos se encuentra el cianuro de potasio, material preferido todavía en los años cincuenta, hasta que los conservadores se hicieron conscientes del peligro que su uso representaba para la salud (Landi 1998:97); fue entonces cuando comenzaron a usar otros quelantes, como la tiourea.

\section{La tiourea}

La tiourea, sulfourea o tiocarbamida es una diamida de ácido tiocarboxílico con una estructura similar a la de la urea, sólo que en el radical ácido tiene azufre en lugar de oxígeno -de allí la inclusión del prefijo -sulfo o -tio-. La característica eléctrica que brinda esta sustitución (valencia -2) y su relativamente pequeño tamaño iónico la hacen muy eficiente en la formación de complejos con iones de plata en condiciones ácidas (Landi 1998:97), pudiendo tener acciones similares con oro y platino (CAMEO 2008). Por ello, las soluciones para limpieza de plata incluyen algún ácido, que suele ser clorhídrico (Stambolov 1966:37-44), sulfúrico, fosfórico o fórmico (Sramek, Jakobsen y Pelikan 1978:114-117) (Figura 1).

Al comparar alrededor de 20 limpiadores comerciales de joyería fue posible verificar que casi todos-soluciones, telas impregnadas o cremas- contienen tiourea. Debido a su rápida acción y uso aparentemente sencillo, son muy aceptados en el mercado.

Al ingresar como parte del personal docente del Seminario Taller de Restauración de Metales (STRM) de la Escuela Nacional de Conservación, Restauración y Museografía (ENCRyM), no cuestioné el empleo

$$
\begin{aligned}
& \mathrm{Ag}_{2} \mathrm{~S}+2 \mathrm{H}^{+}+2 \mathrm{nCS}\left(\mathrm{NH}_{2}\right)_{2} \longrightarrow 2\left[\mathrm{AgCS}\left(\mathrm{NH}_{2}\right)_{2}\right] \mathrm{n}^{+}+\mathrm{H}_{2} \mathrm{~S} \\
& \text { Sulfuro lón Tiourea Tioureato Sulfuro de } \\
& \text { de plata hidrógeno de plata hidrógeno }
\end{aligned}
$$

FIGURA 1. Reacción de la tiourea con sulfuros de plata. 
de la tiourea en la limpieza de plata planteado en el muy famoso y respetado texto de 1971 de Plenderleith y Werner, The Conservation of Antiquities and Works of Art, en el que se aseguraba que sus productos de corrosión podían eliminarse fácilmente con soluciones de limpieza de plata que contenían tiourea al 5 por ciento y un detergente no iónico al 1 por ciento (Plenderleith y Werner 1971:227-229). Si el mismo Plenderleith la recomendaba, se asumía que esta fórmula no tendría efecto secundario alguno para la plata o los recubrimientos de oro, y que su empleo sería seguro.

Aunque la carencia de suficiente registro no permite establecerlo con precisión, en la ENCRyM se empleó durante años una solución de tiourea con las concentraciones descritas por Plenderleith y Werner, acidificada con ácido clorhídrico, posiblemente emulando la composición de uno de los líquidos comerciales limpiadores de plata más empleados, compuesto por agua, tiourea, tensoactivo, ácido clorhídrico y aceite vegetal, cuyo $\mathrm{pH}$ invariablemente es 0 .

Si bien no cuestioné el empleo de tiourea, sí lo hice respecto del ácido clorhídrico, ya que sabía que los iones $\mathrm{Cl}$ son muy dañinos para los metales, pues en presencia de humedad causan corrosión autocatalítica mediante la formación de ácido clorhídrico, y su pequeño tamaño iónico y alta electronegatividad hacen que sean muy difíciles de eliminar; asimismo, la adición de ácido clorhídrico -aunque sea en poca concentración- hace que la solución siempre tenga un $\mathrm{pH} 0$.

Al considerar inadecuado el uso de ácido clorhídrico, busqué opciones y seguí la propuesta de Glenn Wharton, publicada en el Newsletter of the Western Association for Art Conservation (Wharton 1989:4-5), que emplea ácido fosfórico. Aunque el pH seguía siendo muy bajo (1), no pareció del todo inadecuado, pues la plata puede mantenerse estable en ese nivel de acidez $y$, de cualquier forma, se realizaría una neutralización con solución alcalina de carbonato y múltiples enjuagues posteriores con agua y etanol (Wharton 1989:4-5).

Siguiendo esta nueva fórmula, participé de la instrucción de un par de generaciones de restauradores en la limpieza de objetos de plata y plata sobredorada con soluciones de tiourea, con resultados inmediatos muy notables, hasta que en 2006 pude conocer las observaciones que algunos conservadores del Rijksmuseum habían hecho sobre objetos intervenidos años atrás con soluciones de tiourea.

Efectos del uso de tiourea como agente de limpieza

En 2006, en el Metal Conservation Summer Institute, Robert van Langh, conservador del Rijksmuseum, señaló que en este museo se dejó de emplear tiourea como agente limpiador debido a que se había observado que algunas de las obras que años antes se habían limpiado con este material actualmente mostraban superficies deterioradas con microfisuras (Langh 2006).
Desde ese momento comencé a buscar información relativa al empleo y los resultados de este material, la cual fue abundante. Desde 1966, Todor Stambolov (1966:37-44) señalaba que la limpieza con tiourea se dificultaba a causa de la continua formación de sulfuros en las orillas de las zonas tratadas; esto se corregía parcialmente soplando aire para oxidar los iones sulfuro, pero, aun así, las manchas debían corregirse posteriormente con abrasivos.

Según investigaciones relacionadas con la limpieza de daguerrotipos, su acción parece distar de ser controlable, incluso mediante la modificación, para hacerla más selectiva, de las condiciones de $\mathrm{pH}$ (Barger, Krishnaswamy y Messier 1982:13-24). Además, varios autores coinciden en que existe redepositación o reformación de sulfuros (Barger, Giri, White y Edmonson 1986:15-28). También se señala que, pese a su capacidad limpiadora, las soluciones de tiourea alteran la apariencia de las obras, no sólo eliminando la corrosión de la plata, sino llegando a la disolución de la plata metálica, del mercurio, del cobre e incluso del oro, y advierten que el daño es irreversible (Barger, Krishnaswamy y Messier 1982:13-24).

Estudiosos de otros tipos de objetos indican que las superficies de plata tratadas con tiourea tendrán una microrrugosidad característica, que puede describirse como la superficie con apariencia de granos de azúcar obtenida mediante la técnica de grabado al aguafuerte. Este efecto puede eliminarse con un pulimento ligero, pero esto invariablemente constituye un problema para acabados superficiales u objetos de muy poco espesor, como las delgadas láminas que conforman los hilos entorchados.

Quien haya limpiado plata con tiourea sabrá que, si bien en un primer momento las piezas lucen muy limpias, las zonas trabajadas tendrán rápidamente una delgada capa de sulfuros amarillo-marrón que se volverán más oscuros, si no se coloca una capa de protección. Esto puede tener diversas causas, algunas de las cuales se mencionan a continuación.

La reacción de plata y tiourea genera sulfuro de hidrógeno que, en combinación con la humedad del ambiente, se transforma en ácido sulfhídrico. Éste es altamente corrosivo para la plata, y su presencia puede comprobarse por medio del característico olor a huevos podridos, presente durante la aplicación de esta sustancia. Pero aun si no se formara ácido sulfhídrico, el sulfuro de hidrógeno gaseoso en el ambiente puede transformarse en anhídrido sulfuroso y ácido sulfúrico, que generan corrosión tanto en la plata como en el cobre con que se alea.

Por lo anterior, es muy probable que la redepositación de sulfuros registrada desde 1966 por Stambolov se deba menos a la inestabilidad del complejo de tioureato de plata que a una nueva formación de sulfuros, por lo que no es posible evitar el enjuague intensivo de las piezas limpiadas con soluciones de tiourea, para el que se recomienda tallar con detergentes y aclarar por una hora bajo un chorro de agua desionizada para, finalmente, secar con aire, toallas y etanol (Wharton 1989:45). En cualquier caso, este proceso no puede aplicarse, bien 
por sus dimensiones, su estado de conservación o la naturaleza de los materiales que se encuentren asociados, en una gran variedad de objetos que ya se han limpiado con tiourea.

Más aún, investigaciones posteriores han encontrado que el enjuague no ofrece mucha protección, pues se adsorbe tiourea acuosa que produce una película estable de este compuesto, la cual no se remueve con el enjuague $y$, tras el envejecimiento, genera sulfato de diciandiamidina (Kurt 1999:465-466). Aquí se abre una línea de investigación que se ha de desarrollar: por lo pronto, una primera hipótesis es que la tiourea acuosa alojada en poros superficiales del metal, convertida en este compuesto de mayor tamaño y peso molecular (76.12 g/mol-102.09 g/mol), sea responsable de las microfisuras generadas a largo plazo (Figura 2).

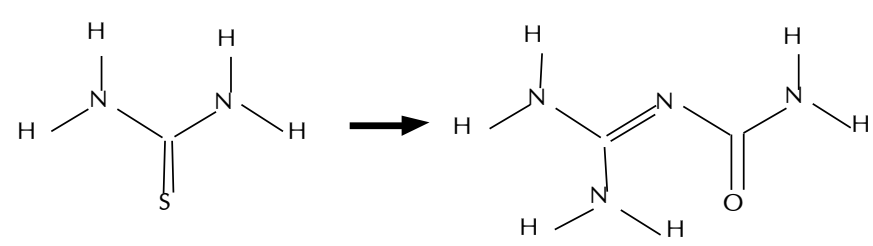

$$
\begin{array}{cc}
\text { Tiourea } & \text { Sulfato de diciandiamidina } \\
\text { Tiocarbamida } & 102.09 \mathrm{~g} / \mathrm{mol} \\
76.12 \mathrm{~g} / \mathrm{mol} &
\end{array}
$$

FIGURA 2. Fórmulas desarrolladas y pesos moleculares de la tiourea y del producto formado por oxidación: sulfato de diciandiamidina.

El panorama parece ser peor en los casos de combinación de materiales, pues, como señala la respetada y conocida Conservation \& Art Material Encyclopedia Online (CAMEO), del Museo de Bellas Artes de Boston, la tiourea puede dañar pintura, coral, marfil, ámbar y una serie de materiales de origen mineral (CAMEO 2008). Posiblemente, esta lista no incluya textiles porque, ante tal posibilidad de alteración, tratarlos con tiourea parece completamente improbable.

Como es bien sabido, la limpieza de hilos entorchados de plata y plata sobredorada siempre ha representado un reto, pues los materiales de limpieza de metales suelen ser muy perniciosos para las fibras textiles. En este caso, es necesario tomar en cuenta, asimismo, que el pH ácido puede alterar aquellos tintes cuya coloración depende de las condiciones de acidez o basicidad, o incluso destruir grupos cromóforos, además de que un $\mathrm{pH}$ tan bajo como 0 invariablemente causará la hidrólisis ácida de fibras tanto proteicas como celulósicas -especialmente si están deterioradas (Timar-Balaszy y Eastop 1998:28, 46, 245)-, pese a que las alteraciones no se noten inmediatamente o incluso demoren algunos pocos años en hacerlo.

Aun aplicando cuidadosamente las soluciones de tiourea con hisopo, resulta imposible impedir que permeen las fibras queconstituyenel almadel hilo, por loque, a largo plazo, el daño en las fibras parece ser inevitable. De esta forma, una inmersión de los hilos en solución de tiourea para eliminar la corrosión estable de la plata es a todas luces injustificable, y acusa no sólo el desconocimiento sobre los materiales, sino una gran irresponsabilidad frente a los bienes culturales cuya restauración se nos ha confiado.

\section{Efectos sobre la salud}

Si la seguridad de los bienes culturales parece lo más relevante, también debe estimarse la de los restauradores. Es conocido que en restauración se emplean muchas sustancias que pueden tener malas repercusiones en la salud. La ignorancia y el exceso de confianza -fundados en el cálculo de que empleamos pocas cantidades- han hecho que durante su utilización adoptemos una actitud hasta cierto punto negligente.

Aunque no se ha comprobado fehacientemente, existen referencias de que la tiourea es potencialmente carcinógena, y, según la ficha de datos de seguridad del producto, que ordena publicar el Reglamento 1907/2006 de la Comunidad Europea, puede causar irritación y quemaduras en la piel y mucosas; náuseas, vómitos y diarreas, si se ingiere; sensibilización alérgica; edema pulmonar por absorción en grandes cantidades, y alteraciones sanguíneas y metabólicas. Adicionalmente, hay que ser muy cuidadosos respecto de los productos secundarios de la reacción: el ácido sulfhídrico se ubica como el más tóxico de los gases, mientras que, entre éstos, el sulfuro de hidrógeno y el anhídrido sulfuroso son sumamente contaminantes.

\section{El inicio de la experimentación}

Si bien la información aquí vertida apunta a que la tiourea no es recomendable para la limpieza de objetos de plata -de modo que incluso resulta sorprendente que, con el único sustento, un tanto frívolo, de que se obtiene una mejor apariencia de los objetos al culminar la intervención, aún haya quienes defienden su empleo en procesos de restauración profesional-, sería asimismo irresponsable no realizar la investigación necesaria para comprobar que esta sustancia resulta verdaderamente perniciosa.

Por lo pronto, tal investigación, que debe identificar la obtención de superficies microrrugosas, la remanencia de tiourea adsorbida en los microporos de los objetos de plata, la formación de sulfato de diciandiamidina y la generación de microfisuras, la he planteado como tema de tesis de la Maestría en Ciencias en Conservación Forense. En tanto pueda llevarla a cabo, para este texto realicé el siguiente pequeño experimento, sólo como un acercamiento al problema.

Se emplearon diez probetas, consistentes en monedas de plata ley 0.720, que se pulieron, lavaron, enjuagaron con agua destilada y alcohol etílico. Cinco de éstas fueron doradas electroquímicamente; ocho, corroídas durante treinta minutos con una solución de sulfuro de amonio al 3 por ciento. La capa de sulfuro de plata así lograda resultó compacta -aunque no del todo homogénea- en las piezas sobredoradas. Se mantuvieron testigos: una moneda 
de plata y una sobredorada limpias, y dos, de las mismas características, corroídas.

Las seis probetas restantes se limpiaron de la siguiente forma:

- Solución comercial, "Limpiasteg 130", de tiourea con ácido clorhídrico. Se verificó su pH en 0 y la limpieza se hizo por inmersión, de acuerdo con las instrucciones incluidas. Se requirieron dos minutos para dejarlas completamente limpias. - Solución de tiourea al 5 por ciento en solución de ácido fosfórico al 8 por ciento v/v (Wharton 1989:4-5). El pH de la solución fue 1. La limpieza se llevó a cabo por inmersión durante cinco minutos.

- Limpieza electroquímica. Por inmersión en una solución de carbonato de sodio al 10 por ciento $\mathrm{p} / \mathrm{v}$ como electrolito y usando aluminio como ánodo, durante dos horas para la moneda de plata y treinta minutos para la sobredorada. Esta limpieza se hizo sólo para descartar que la corrosión previa fuera la causante de las rugosidades $y$, por lo tanto, se presentara en cualquier superficie tratada.

Después de cada limpieza, todas las probetas se enjuagaron en agua corriente, agua destilada y alcohol etílico, y se secaron con algodón para después analizarse bajo microscopio estereoscópico (Figura 3).

\section{Resultados}

Solución comercial de tiourea con ácido clorhídrico

La moneda de plata se limpió rápida y totalmente, incluso se eliminaron aquellos productos de corrosión que permitían distinguir sus detalles. En la probeta de plata sobredorada también se eliminaron todos los productos de corrosión, incluidos los que estaban debajo de la capa de oro, por lo que hubo pérdida de dorado. En ambos casos es evidente la superficie microrrugosa. Pasada una semana, ambas monedas presentaron zonas con una ligera capa de corrosión marrón -sulfuros-, y algunos puntos más gruesos en la base de las orlas y gráfilas de la moneda.

Tiourea en solución de ácido fosfórico

Tras cinco minutos de inmersión, esta solución generó en la moneda de plata una limpieza homogénea, aunque una
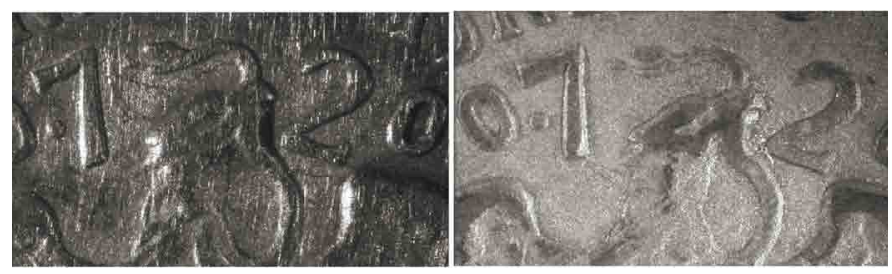

FIGURA 3. Detalles de las superficies de las probetas de plata sobredorada vistas al microscopio estereoscópico; la de la izquierda, limpia; la de la derecha, corroída. (Cortesía de la autora.) pequeña zona conservó una capa de corrosión. Por su parte, la superficie sobredorada se limpió de manera heterogénea: se mantuvo un alto porcentaje de corrosión, que impidió apreciar el dorado. Si bien algunos productos de corrosión no se eliminaron, los detalles de las dos monedas son más legibles que en el caso anterior, y la microrrugosidad, menos evidente. Pasada una semana, no se apreciaron mayores cambios en las superficies.

\section{Limpieza electroquímica}

Se ha comprobado que la limpieza electroquímica no representa riesgo para la plata, y puede repetirse hasta lograr el resultado deseado, lo que ocurrió aquí, pues ninguna de las monedas presenta microrrugosidades. Incluso se diría que la limpieza permitió conservar los productos de corrosión que, al acentuar convenientemente los detalles, eventualmente se considerarían pátina. Pese a ello, este método de limpieza no se considera adecuado para plata sobredorada, ya que la cavitación, causada por la producción de hidrógeno, característica de esta técnica, causó pérdida del dorado, efecto inaceptable en un bien cultural. Tras una semana, ninguna de las monedas tratadas con este método presenta cambios.

\section{Conclusiones}

Es un hecho que las soluciones de tiourea son realmente efectivas en la eliminación de productos de corrosión de plata, y no se puede negar que las soluciones comerciales son, además, muy rápidas, pero la información -disponible desde hace décadas- sobre los efectos negativos de su uso como agente de limpieza de plata es abundante y accesible, $y$, por ende, resulta lamentable que no la conociéramos aquí, por actuar como si la restauración fuera apenas un oficio, un arte mecánica, e irreflexiva, que sólo exige elegir la fórmula.

Hemos usado muchos materiales por simple hábito (la tiourea constituye sólo un ejemplo de tantos). No nos cuestionamos mayormente sobre sus consecuencias, mecanismos de acción, interacciones, productos secundarios, etc., lo que sin duda ha llevado a cometer más de un error de intervención y, no esporádicamente, a actitudes poco científicas y constructivas, con argumentos del tipo así se ha usado antes y ha quedado bien o, peor aún, si yo no lo he visto, no existe.

Un profesional de la restauración informado y consciente no puede sugerir tiourea ni cualquier otro agente sin el respaldo de evaluaciones analíticas y experimentales repetibles. Si bien se mantiene la inercia de seguir considerando a la restauración como un oficio, un mero trabajo manual, y aún no contamos con una estructura institucional que promueva y facilite que constituyamos verdaderos grupos de investigación en torno de materiales y procesos de restauración que permitan seguimientos estandarizados y análisis sobre los resultados de nuestro trabajo en el largo plazo, es nuestra responsabilidad -de los restauradores profesiona- 


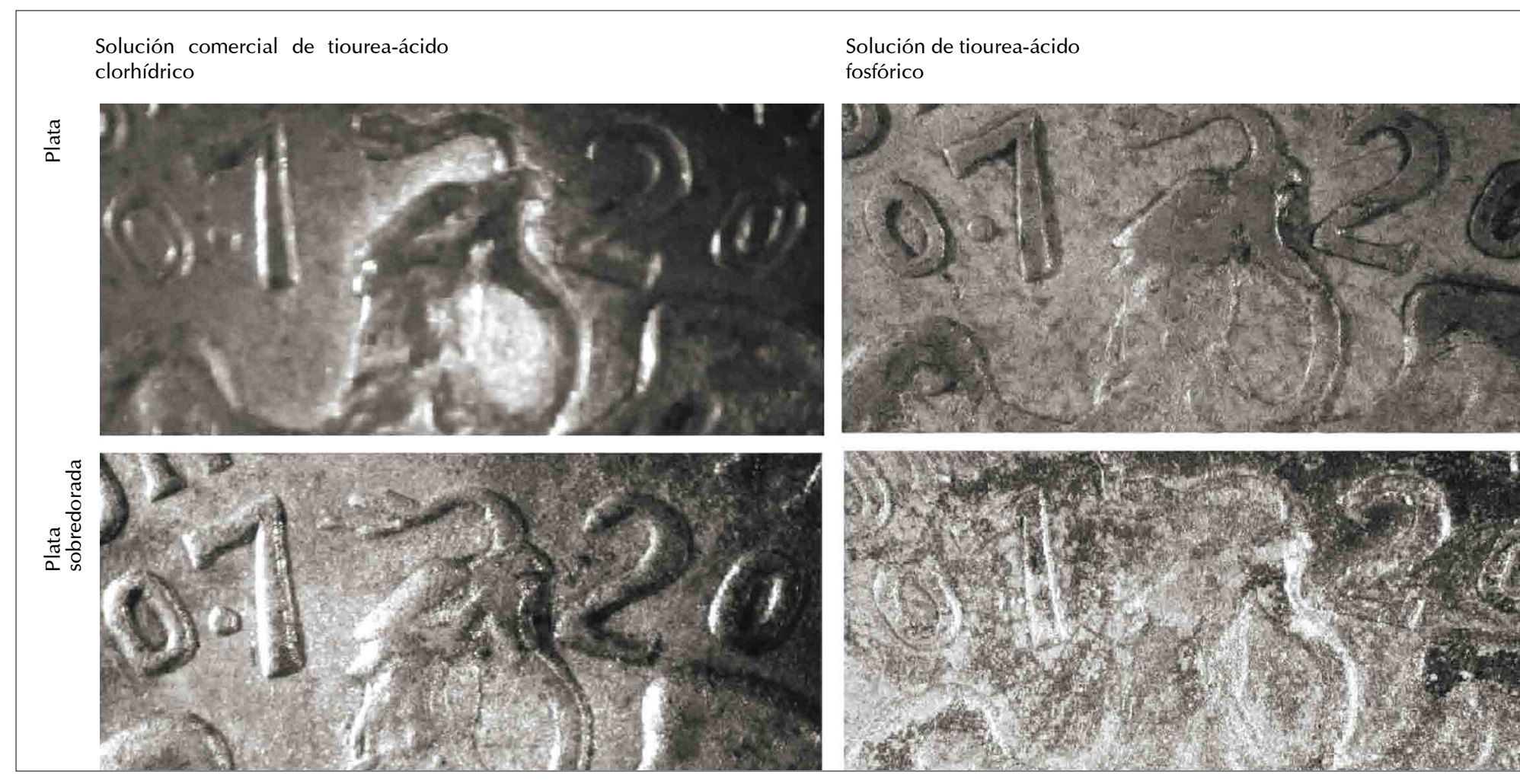

FIGURA 4. Resultados de la limpieza: con las dos soluciones de tiourea y electrolítica. (Cortesía de la autora.)

les- hacer que esto suceda. Debemos dejar de actuar como si Plenderleith hubiera resuelto todo en un solo libro, como si todas las preguntas ya se hubieran planteado y respondido de la única forma posible.

Entre nosotros hay, por supuesto, muchos profesionales conscientes que, pese a las resistencias del exterior, se atreven a cuestionar, analizar y proponer opciones, rompiendo así el anquilosamiento y el hábito, y dan carácter de ciencia y de arte a esta profesión, que sólo así podrá mejorar y ser verdaderamente útil. Nuestra tarea es formar a muchos más de éstos.

\section{Referencias}

Barger, Susan, A. P. Giri, William B. White y Thomas M. Edmondson 1986 "Cleaning Daguerreotypes", Studies in Conservation 31 (1): 15-28.

Barger, M. Susan, S. V. Krishnaswamy y Russell Messier 1982 "The Cleaning of Daguerreotypes: Comparison of Cleaning Methods", Journal of the American Institute of Conservation 22 (1): 13-24.

Baez Vicke, Margarita, et al. 2007 "Informe de los trabajos de restauración realizados a la colección del Museo Nacional de Historia, vajilla Christofle y un revólver", documento mecanoescrito del Seminario Taller de Metales, Escuela Nacional de Conservación, Restauración y Museografía, México, ENCRyM-INAH.

CAMEO

2008 Conservation \& Art Material Encyclopedia Online, Museum of Fine Arts, Boston, documento electrónico disponible en http://cameo.mfa.org, consultado en mayo de 2008.

Chemicalland

2008 Thiourea (Thiocarbamide), AroKor, Holdings, documento electrónico disponible en http://chemicalland21.com/industrialchem/organic/THIOUREA.htm, consultado en mayo de 2008.

Kurth, Dirk

1999 "The Reaction of Thiourea to Dicyandiamidine Sulfate on Silver Surfaces Investigated by Reflection-Absorption Infrared Spectroscopy", Fresenius' Journal of Analytical Chemistry 365 (5): 465-466.

Landi, Sheila

1992 The Textile Conservator's Manual, Londres, ButterworthHeinemann.

Plenderleith, Herbert y Alfred Emil A. Werner

1971 The Conservation of Antiquities and Works of Art: Treatment, Repair, and Restoration, Oxford, Oxford University Press.

ReCollections

2004 Caring for Cultural Material, documento electrónico dis- 
Limpieza electroquímica
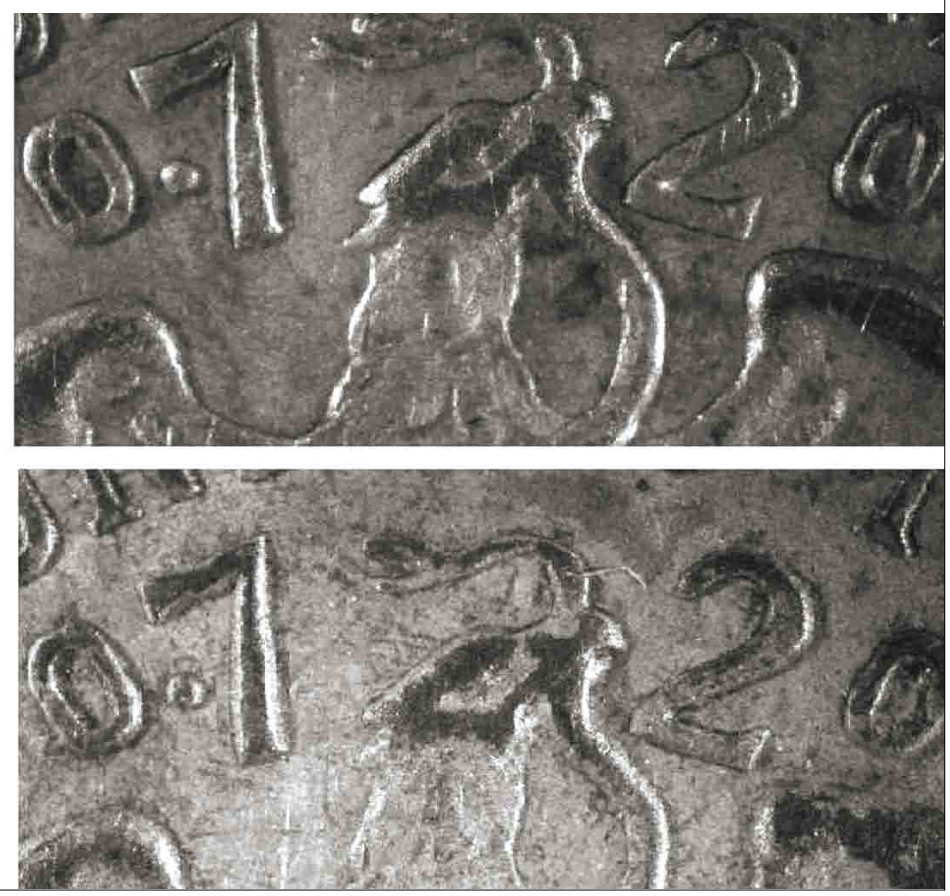

ponible en http://amol.org.au/recollections/2/5/03.htm, consultado en agosto de 2004.

Rudoe, Judy

1993 "Oxidized Silverinthe Nineteenth Century: The Documentary Evidence", en Susan La Niece y Paul Craddock (eds.), Metal Plating and Patination: Cultural, Technical and Historical Developments, Londres, Butterworth-Heinemann, 161-170.

Šramek, Jirí, Tove B. Jakobsen y Jirí B. Pelikan

1978 "Corrosion and Conservation of a Silver Visceral Vessel From the Beginning of the Seventeenth Century", Studies in Conservation 23 (3): 114-117.

Stambolov, Todor

1966 "Removal of Corrosion on an Eighteenth-Century Silver Bowl", Studies in Conservation 11 (1): 37-44.

The PubChem Project

2008 The PubChem Project, documento electrónico disponible en http://pubchem.ncbi.nlm.nih.gov, consultado en mayo de 2008.

Tímar-Bálázsy, Ágnes y Dinah Eastop

1998 Chemical Principles of Textile Conservation, Londres, Butterworth-Heinemann.

Wharton, Glenn

1989 "The Cleaning and Lacquering of Museum Silver", Newsletter of the Western Association of Art Conservation 11 (1): 4-5.

\section{Resumen}

El uso de la tiourea para la limpieza de bienes culturales en plata no parece totalmente conveniente. Además de poseer un $\mathrm{pH} 0$, derivado de la presencia de ácido clorhídrico, que afecta la conservación del material constitutivo, la bibliografía indica que sus residuos no pueden ser eliminados por completo, que forma productos secundarios, y que es posible que se generen microfisuras superficiales. El presente artículo evalúa este tratamiento, no sólo desde el punto de vista técnico, sino desde el ético. Así, se critica su uso como un ejemplo de la aplicación de materiales que, por mero hábito -sin investigación sobre sus consecuencias, sus mecanismos de acción, sus interacciones, sus productos secundarios, etc.- han llevado a errores en la intervención. Adicionalmente, se reflexiona sobre la actitud profesional que puede posicionar a la restauración como un oficio: una actividad que prioriza la habilidad manual y la aplicación de fórmulas sobre la reflexión y la investigación. Se concluye que un pensamiento científico es indispensable para el desarrollo de la disciplina.

\section{Palabras clave}

Tiourea, ciencia, oficio, restauración, investigación.

\section{Abstract}

The use of thiourea for the cleaning of silver cultural heritage has not been absolutely convenient. Apart from having a $0-\mathrm{pH}$, as a result from the presence of clorhidric acid, which affects the conservation of the constituent materials, the bibliography indicates that its residues cannot be completely eliminated, that it forms secondary by-products, and that it might cause superficial micro-fissures. Hence, this article critics its use, as an example of the application of materials, that by habit -without investigating its consequences, its mechanisms of action, its interactions, and its secondary by-products- have lead to serious mistakes in the intervention. It additionally analyses the professional attitude that can place restoration as an art-craft: an activity that prioritizes manual skills and the application of formulas over the self-reflection and investigation. It concludes that a scientific thinking is indispensable for the discipline development.

\section{Keywords}

Thiourea, Science, Office, Restoration, Investigation. 